Bull. Austral. Math. Soc.

VoL. $62(2000) \quad[435-443]$

\title{
ASYMPTOTIC BEHAVIOUR OF SMALL SOLUTIONS OF SINGULARLY PERTURBED PROBLEMS
}

\section{ZONGMING GuO}

We consider the singular limit of small solutions of some singularly perturbed problems where the nonlinearity changes sign.

\section{INTRODUCTION}

In this paper we consider the singular limit of positive solutions of

$$
-\varepsilon^{2} \Delta u=f(u) \text { in } B, \quad u=0 \text { on } \partial B .
$$

Here $B$ is the unit ball in $\mathbf{R}^{N}(N>2), \varepsilon>0$. We are interested in the asymptotic behaviour of positive solutions for small positive $\varepsilon$ in the case that $f(0)=0, f^{\prime}(0)=0$ and $f$ changes sign on $[0, \infty)$. More precisely, we assume $f \in C^{1+\sigma}((0, \infty)) \cap C^{0}([0, \infty))$ with $0<\sigma<1$ satisfying the following conditions:

$\left(f_{1}\right) \quad f(0)=0, f$ has two positive zeros $a$ and $b$ such that $a<b, f^{\prime}(b)<0$, and $f$ has no other positive zeros;

$\left(f_{2}\right) \quad(s-a) f^{\prime}(s)<f(s)$ on $(a, b)$, and there is a $\rho>N(N-2)^{-1}$ such that $\lim _{s \rightarrow 0^{+}} s^{1-\rho} f^{\prime}(s)$ exists and is negative;

$\left(f_{3}\right) \quad \int_{0}^{b} f(s) d s>0$ and $\beta$ is the unique number in $(a, b)$ such that $\int_{0}^{\beta} f(s) d s=0$.

Problem (1.1) has appeared in various models in applied mathematics, including population genetics and chemical reactor theory (see, for example [7] and the references therein) and has been studied by many authors, see, for example, $[9,10,4,7]$. The case that $f^{\prime}(0)=0$ can be viewed as a border line case of singular perturbation problems (see [6]). Benci and Cerami [1] raised the question what happens for the structure of positive solutions in this borderline case, also called the zero mass case [2].

In [7], Jang studied the problem similar to $(1.1)$, but with $f^{\prime}(0)<0$ and a general smooth domain $\Omega$. He found a positive small solution $u_{\varepsilon}$ of (1.1), which has only one maximum point $P_{\varepsilon} \in \Omega$ when $\Omega$ is a convex domain. In [10], under the same conditions

Received 28th February, 2000

Copyright Clearance Centre, Inc. Serial-fee code: 0004-9727/00 \$A2.00+0.00. 
as in [7], Ni, Takagi and Wei showed that $v_{\varepsilon}(y):=u_{\varepsilon}\left(\varepsilon y+P_{\varepsilon}\right) \rightarrow V$ as $\varepsilon \rightarrow 0$ in $C_{l o c}^{2}\left(\mathbf{R}^{N}\right)$ where $V=V(x)$ is the unique positive (radial) solution of

$$
\Delta V+f(V)=0 \text { in } \mathbf{R}^{N}, \quad V(x) \rightarrow 0 \text { as }|x| \rightarrow \infty
$$

and

$$
\lim _{\varepsilon \rightarrow 0} \operatorname{dist}\left(P_{\varepsilon}, \partial \Omega\right)=\max _{P \in \Omega} \operatorname{dist}(P, \partial \Omega) .
$$

To obtain the mentioned results, the authors always use the fact that $V$ decays exponentially as $|x| \rightarrow \infty$ (since $f^{\prime}(0)<0$ ). Meanwhile, Dancer [4] studied (1.1) in a domain $D$ of type $R_{N}$ with $f^{\prime}(0)=0$. He showed that when $f$ satisfies $\left(f_{1}\right)-\left(f_{3}\right),(1.1)$ has exactly 2 positive solutions $\bar{u}_{\varepsilon}, \underline{u}_{\varepsilon}$ with $0<\|u\|_{\infty}<b$ for all small positive $\varepsilon: \bar{u}_{\varepsilon}$ is a large solution, that is, $\bar{u}_{\varepsilon} \rightarrow b$ uniformly on compact subsets of $D$ as $\varepsilon \rightarrow 0 ; \underline{u}_{\varepsilon}$ is a small solution, that is, $\left\|\underline{u}_{\varepsilon}\right\|_{\infty}<b$ and $v_{\varepsilon}(y):=\underline{u}_{\varepsilon}(\varepsilon y) \rightarrow V$ as $\varepsilon \rightarrow 0$ in $C_{\text {loc }}^{2}\left(\mathbf{R}^{N}\right)$ where $V=V(x)$ is the unique positive (radial) solution of

$$
\Delta V+f(V)=0 \text { in } \mathbf{R}^{N}, \quad V^{\prime}(|x|)<0, \quad V(x) \rightarrow 0 \text { as }|x| \rightarrow \infty
$$

We easily see that the conclusions of [4] are definitely true on the unit ball. In this paper we shall analyse the exact behaviour of $\underline{u}_{\varepsilon}$ as $\varepsilon \rightarrow 0$. Notice that the arguments in $[\mathbf{7}, \mathbf{1 0}]$ can not be used here, since in our case $f^{\prime}(0)=0$. The main difficulty is that in our case, the decay rate of the unique positive (radial) solution of the problem (1.4) obtained in [4] is not exponential.

\section{AN UPPER BOUND FOR $\underline{u}_{\varepsilon}$}

In this section we exhibit a property of $\underline{u}_{\varepsilon}$, in that it can be globally bounded by means of a function $K \varepsilon^{N-2}|x|^{2-N}$ for $\varepsilon$ sufficiently small and some appropriately chosen constants $K>0$ independent of $\varepsilon$. We consider the problem along some subsequences of $\varepsilon$.

By [5], we know that $\underline{u}_{\varepsilon}$ is a radial function and $\underline{u}_{\varepsilon}^{\prime}(r)<0$ for $r \in(0,1]$. Therefore, $\underline{u}_{\varepsilon}$ satisfies the problem

$$
-\left(r^{N-1} \underline{u}_{\varepsilon}^{\prime}\right)^{\prime}=r^{N-1} \varepsilon^{-2} f\left(\underline{u}_{\varepsilon}\right) \text { in }(0,1), \quad \underline{u}_{\varepsilon}^{\prime}(0)=0, \underline{u}_{\varepsilon}(1)=0 .
$$

THEOREM 2.1. Let $\underline{u}_{\varepsilon}$ be the small solution of problem (2.1). Then there exists $\varepsilon_{0}>0$ such that for $0<\varepsilon<\varepsilon_{0}$

$$
\underline{u}_{\varepsilon}(r) \leqslant K \varepsilon^{N-2} r^{2-N} \text { for } r \in(0,1)
$$

where $K>0$ is independent of $\varepsilon$. 
PRoOF: It follows from [4] that for $\varepsilon$ sufficiently small,

$$
\max \underline{u}_{\varepsilon}=\underline{u}_{\varepsilon}(0)>\beta .
$$

Define $r_{1}=r_{1}(\varepsilon), r_{2}=r_{2}(\varepsilon) \in(0,1)$ such that

$$
\underline{u}_{\varepsilon}\left(r_{1}\right)=a, \quad \underline{u}_{\varepsilon}\left(r_{2}\right)=a / 2 .
$$

Making the changes of variables:

$$
y=\varepsilon^{-1} r, \quad v_{\varepsilon}(y)=\underline{u}_{\varepsilon}(r)
$$

we have that $v_{\varepsilon}$ satisfies the problem

$$
-\left(y^{N-1} v_{\varepsilon}^{\prime}\right)^{\prime}=y^{N-1} f\left(v_{\varepsilon}\right) \text { in }\left(0, \varepsilon^{-1}\right), \quad v_{\varepsilon}\left(\varepsilon^{-1}\right)=0 .
$$

(Note that $y$ depends upon $\varepsilon$.) It follows from [4] that there exists a subsequence of $\left\{v_{\varepsilon}\right\}$ (still denoted by $\left\{v_{\varepsilon}\right\}$ ) such that

$$
v_{\varepsilon}(y) \rightarrow V \text { in } C_{l o c}^{1}(0, \infty) \text { as } \varepsilon \rightarrow 0
$$

Since $\underline{u}_{\varepsilon}$ is decreasing, then $v_{\varepsilon}$ is decreasing on $\left(0, \varepsilon^{-1}\right)$. Set $y_{1}=y_{1}(\varepsilon)=\varepsilon^{-1} r_{1}, y_{2}=$ $y_{2}(\varepsilon)=\varepsilon^{-1} r_{2}$. Then

$$
v_{\varepsilon}\left(y_{1}\right)=a, \quad v_{\varepsilon}\left(y_{2}\right)=a / 2
$$

and

$$
y_{1} \rightarrow Y_{1}, \quad y_{2} \rightarrow Y_{2} \text { as } \varepsilon \rightarrow 0
$$

where $Y_{1}, Y_{2} \in(0, \infty)$ satisfies

$$
V\left(Y_{1}\right)=a, \quad V\left(Y_{2}\right)=a / 2
$$

On the other hand, it easily follows from (2.3) that $y^{N-1} v_{\varepsilon}^{\prime}(y)$ is increasing in $\left(y_{1}, \varepsilon^{-1}\right)$ (since $v_{\varepsilon}<a$ in $\left(y_{1}, \varepsilon^{-1}\right)$ ). Thus,

$$
y^{N-1} v_{\varepsilon}^{\prime}(y)>y_{1}^{N-1} v_{\varepsilon}^{\prime}\left(y_{1}\right) \text { for } y \in\left(y_{1}, \varepsilon^{-1}\right) .
$$

Now we show that there exists $A>0$ independent of $\varepsilon$ such that

$$
y_{1}^{N-1} v_{\varepsilon}^{\prime}\left(y_{1}\right)>-A
$$

Since $v_{\varepsilon} \rightarrow V$ in $C_{l o c}^{1}(0, \infty)$, we easily obtain that

$$
v_{\varepsilon}^{\prime}(y) \rightarrow V^{\prime}(\widetilde{y}) \text { for } y \in\left[y_{1}, y_{2}\right] \text { as } \varepsilon \rightarrow 0,
$$


where $\tilde{y}=\lim _{\varepsilon \rightarrow 0} y$. It is easily shown that there exists $B>0$ independent of $\varepsilon$ such that $V^{\prime}(\tilde{y})>-B$ for $\tilde{y} \in\left[Y_{1}, Y_{2}\right]$. This implies that there exists $\varepsilon_{0}>0$ such that for $0<\varepsilon<\varepsilon_{0}$,

$$
v_{\varepsilon}^{\prime}(y)>-(B+1) \text { for } y \in\left[y_{1}, y_{2}\right]
$$

Therefore, (2.8) can be obtained by the fact that $y_{1}^{N-1} \rightarrow Y_{1}^{N-1}$ as $\varepsilon \rightarrow 0$. (2.7) and (2.8) imply that

$$
v_{\varepsilon}^{\prime}(y) \geqslant-A y^{1-N} \text { for } y \in\left[y_{1}, \varepsilon^{-1}\right)
$$

Integrating $(2.10)$ on $\left(y, \varepsilon^{-1}\right)$, we obtain that

$$
v_{\varepsilon}(y) \leqslant \frac{A}{N-2} y^{2-N} \text { for } y \in\left[y_{1}, \varepsilon^{-1}\right) .
$$

Since $v_{\varepsilon}(y) \rightarrow V(\widetilde{y})$ for any $y \in\left[0, y_{1}\right]$ and $\tilde{y} \in\left[0, Y_{1}\right]$, it follows that there exists $C>0$ such that for $\varepsilon \in\left(0, \varepsilon_{0}\right)$,

$$
v_{\varepsilon}(y) \leqslant C y^{2-N} \text { for } y \in\left[0, y_{1}\right] .
$$

(2.11) and (2.12) imply our conclusion by choosing $K=\max \{A /(N-2), C\}$.

\section{ASYMPTOTIC BEHAVIOUR OF $\underline{u}_{\varepsilon}$}

In this section we turn to a description of the small solution $\underline{u}_{\varepsilon}(x)$ defined in Theorem 2.1 as $\varepsilon \rightarrow 0$. Thus, we consider the problem below along a subsequence of $\varepsilon$. As in [3] and [8] the Pohozaev Identity plays a central role here. For the small solution $\underline{u}_{\varepsilon}$ of (1.1) it becomes

$$
N \int_{B} F\left(\underline{u}_{\epsilon}\right)-\frac{N-2}{2} \int_{B} \underline{u}_{\varepsilon} f\left(\underline{u}_{\varepsilon}\right)=\frac{\varepsilon^{2}}{2} \int_{\partial B}(x-y, n)\left(\frac{\partial \underline{u}_{\varepsilon}}{\partial n}\right)^{2},
$$

where $F(u)=\int_{0}^{u} f(s) d s, y$ any point in $\mathbf{R}^{N}$ and $n$ the outward pointing normal vector on $\partial B$. This yields as a consequence, with $y=0$,

$$
N \int_{B} F\left(\underline{u}_{\varepsilon}\right)-\frac{N-2}{2} \int_{B} \underline{u}_{\varepsilon} f\left(\underline{u}_{\varepsilon}\right)=\frac{\varepsilon^{2}}{2} \int_{\partial B}(x, n)\left(\frac{\partial \underline{u}_{\varepsilon}}{\partial n}\right)^{2},
$$

and, upon differentiation with respect to $y$,

$$
\int_{\partial B}\left(\frac{\partial \underline{u}_{\varepsilon}}{\partial n}\right)^{2} n=0 \text {. }
$$

The main result in this section is the following theorem:

THEOREM 3.1. Let $f$ satisfy $\left(f_{1}\right)-\left(f_{3}\right)$ and let $\underline{u}_{\varepsilon}$ be the small solution of problem (1.1). Then

$$
\varepsilon^{2-N} \underline{u}_{\varepsilon}(x) \rightarrow J G_{0}(x) \text { as } \varepsilon \rightarrow 0
$$


where $G_{0}=G(\cdot, 0)$ is the Green's function of $-\Delta$ with zero Dirichlet boundary conditions in $B$,

$$
J=\int_{\mathbf{R}^{N}} f(V(y)) d y
$$

$V(y)$ is the unique positive (radial) solution of (1.4). Moreover,

$$
N \int_{\mathbf{R}^{N}} F(V(y)) d y=\frac{N-2}{2} \int_{\mathbf{R}^{N}} V(y) f(V(y)) d y
$$

To prove this theorm, some basic elliptic estimates will be needed. They are supplied by the following lemma and corollary which we take from [3] and [8].

LEMMA 3.2. Suppose $u$ is the solution of the problem

$$
-\Delta u=g \text { in } \Omega, \quad u=0 \text { on } \partial \Omega,
$$

where $\Omega$ is a bounded domain in $\mathbf{R}^{N}$ with smooth boundary $\partial \Omega$. Then there is a constant $C>0$, which depends only on $\Omega$, such that

$$
\|u\|_{W^{1, s}(\Omega)}+\|\nabla u\|_{C^{0, \alpha}(\partial \Omega)} \leqslant C\left(\|g\|_{L^{1}(\Omega)}+\|g\|_{L^{\infty}(\omega)}\right)
$$

for any $s<N /(N-1)$, any $\alpha \in(0,1)$ and any neighbourhood $\omega$ of $\partial \Omega$.

Corollary 3.3. In the notation of Lemma 3.2, we have for $1<m<s N /(N-s)$,

(a) $\|u\|_{L^{m}(\Omega)}+\|\nabla u\|_{L^{2}(\partial \Omega)} \leqslant C\left(\|g\|_{L^{1}(\Omega)}+\|g\|_{L^{\infty}(\omega)}\right)$.

(b) If $\left\{g_{n}\right\}$ is a bounded sequence in $L^{1}(\Omega)$ and in $L^{\infty}(\omega)$, then the corresponding sequence of solutions $\left\{u_{n}\right\}$ has compact closure in $L^{m}(\Omega)$, whilst the sequence $\left\{\nabla u_{n}\right\}$, restricted to $\partial \Omega$, has compact closure in $L^{2}(\partial \Omega)$.

Now we introduce the rescaled variables:

$$
y=\varepsilon^{-1} x, \quad v_{\varepsilon}(y)=\underline{u}_{\varepsilon}(x)
$$

to determine the behaviour of $\underline{u}_{\varepsilon}$ near the origin.

LEMMA 3.4. We have

$$
v_{\varepsilon}(y) \rightarrow V \text { as } \varepsilon \rightarrow 0
$$

uniformly on $\mathbf{R}^{N}$, where $V$ is the unique radially symmetric solution of the problem (1.4). Moreover,

$$
V(y)=0\left(|y|^{-(N-2)}\right) \text { as }|y| \rightarrow \infty .
$$

ProOF: Because the family $\left\{v_{\varepsilon}\right\}$ is uniformly bounded in $\mathbf{R}^{N}$, it follows from elliptic regularity theory that there exists a sequence, also denoted by $\left\{v_{\varepsilon}\right\}$, which converges uniformly on compact sets to the unique positive radial solution of (1.4). It follows from 
the proof of Theorem 2.1 that there is a constant $K>0$ which does not depend upon $\varepsilon$ such that

$$
v_{\varepsilon}(y) \leqslant K|y|^{-(N-2)} \text { in } \mathbf{R}^{N}
$$

for $\varepsilon$ small enough. This implies that the convergence of $v_{\varepsilon}$ to $V$ is actually uniform in the whole $\mathbf{R}^{N}$ and that

$$
V(y) \leqslant K|y|^{-(N-2)} \text { for } y \in \mathbf{R}^{N} .
$$

For future reference we note the following limit.

LEMMA 3.5 .

$$
\lim _{\varepsilon \rightarrow 0} \varepsilon^{-N} \int_{B} f\left(\underline{u}_{\varepsilon}\right) d x=\int_{\mathbf{R}^{N}} f(V(y)) d y .
$$

Proof: Transforming to the variables $y$ and $v_{\varepsilon}$, we obtain

$$
\varepsilon^{-N} \int_{B} f\left(\underline{u}_{\varepsilon}(x)\right) d x=\int_{B_{\varepsilon-1}} f\left(v_{\varepsilon}(y)\right) d y .
$$

For $\varepsilon$ sufficiently small, it follows from (3.3) that

$$
v_{\varepsilon}(y) \leqslant \widehat{v}(y)=\min \left\{b, K|y|^{-(N-2)}\right\} .
$$

Since $|f(s)| \leqslant \theta s^{\rho}$ for $s \in(0, b)$ with some $\theta>0$ and $\rho>N(N-2)^{-1}$, then $\hat{v} \in L^{\rho}\left(\mathbf{R}^{N}\right)$ and it follows from Lemma 3.4 and the dominated convergence theorem that

$$
\int_{B_{e^{-1}}} f\left(v_{\varepsilon}(y)\right) d y \rightarrow \int_{\mathbf{R}^{N}} f(V(y)) d y
$$

from which the assertion follows.

In what follows we shall write

$$
J=\int_{\mathbf{R}^{N}} f(V(y)) d y .
$$

The limiting behaviour of the left-hand side of the Pohozaev Identity (3.1) now readily follows from Lemma 3.5:

$$
\begin{aligned}
\lim _{\varepsilon \rightarrow 0} \varepsilon^{-N}\left(N \int_{B} F\left(\underline{u}_{\varepsilon}\right)-\frac{N-2}{2} \int_{B} \underline{u}_{\varepsilon} f\left(\underline{u}_{\varepsilon}\right)\right) & \\
& =N \int_{\mathbf{R}^{N}} F(V(y)) d y-\frac{N-2}{2} \int_{\mathbf{R}^{N}} V(y) f(V(y)) d y .
\end{aligned}
$$

Proof OF THEOREM 3.1

We define the function $g(x, y)$ which is the regular part of the Green's function $G(x, y)$ which solves

$$
-\Delta G=\delta_{y} \text { in } B, \quad G=0 \text { on } \partial B
$$


and is given by the relation

$$
G(x, y)=\frac{1}{(N-2) \sigma_{N}|x-y|^{N-2}}+g(x, y),
$$

where $\sigma_{N}$ is the area of the unit sphere in $\mathbf{R}^{N}$. To determine the behaviour of $\underline{u}_{c}$ away from the origin and to estimate the right-hand side of (3.1) we define the function

$$
w_{\varepsilon}(x)=\epsilon^{2-N} \underline{u}_{\varepsilon}(x) .
$$

By $(1.1), w_{\varepsilon}$ is as solution of the problem

$$
-\Delta w_{\varepsilon}=h_{\varepsilon}(x) \text { in } B, \quad w_{\varepsilon}=0 \text { on } \partial B
$$

where

$$
h_{\varepsilon}(x)=\varepsilon^{-N} f\left(\underline{u}_{\varepsilon}\right) .
$$

According to Theorem 2.1, (3.7) and the conditions on $f$, we have for $x \neq 0$,

$$
\left|h_{\varepsilon}(x)\right| \leqslant C \varepsilon^{-N} \underline{u}_{\varepsilon}^{\rho} \leqslant C(N(N-2))^{(N-2) / 2} \varepsilon^{-N+\rho(N-2)}|x|^{(2-N) \rho}
$$

and so, if $x \neq 0$, then

$$
h_{\varepsilon}(x) \rightarrow 0 \text { as } \varepsilon \rightarrow 0
$$

since $\rho>N(N-2)^{-1}$.

On the other hand,

$$
\int_{B} h_{\varepsilon}(x) d x=\varepsilon^{-N} \int_{B} f\left(\underline{u}_{\varepsilon}\right) d x
$$

Hence, by Lemma 3.5

$$
\lim _{\varepsilon \rightarrow 0} \int_{B} h_{\varepsilon}(x) d x=J
$$

From (3.8) and (3.9) we conclude that

$$
h_{\varepsilon} \rightarrow J \delta_{0} \text { as } \varepsilon \rightarrow 0,
$$

where $\delta_{0}$ is the Dirac mass centred at the origin. This implies, according to Corollary 3.3 , that

$$
w_{\varepsilon} \rightarrow J G_{0} \text { as } \varepsilon \rightarrow 0
$$

in $L^{m}(B)$, as well as in $L^{\infty}(\omega)$, where $\omega$ is any compact subset of $B$ which does not contain the origin. Here $G_{0}=G(\cdot, 0)$, where $G$ is the Green's function of $-\Delta$ with zero Dirichlet boundary conditions in $B$. It is given by

$$
G_{0}(x)=\frac{1}{(N-2) \sigma_{N}}\left(\frac{1}{|x|^{N-2}}-1\right)
$$


In addition we conclude from Corollary 3.3 that on the boundary $\partial B$

$$
\nabla w_{\varepsilon} \rightarrow J \nabla G_{0} \text { as } \varepsilon \rightarrow 0 \text { in } L^{2}(\partial B)
$$

This yields for the right-hand side of (3.1)

$$
\varepsilon^{2(2-N)} \int_{\partial B}(x, n)\left(\frac{\partial \underline{u}_{\varepsilon}}{\partial n}\right)^{2} \rightarrow J^{2} \int_{\partial B}(x, n)\left(\frac{\partial G_{0}}{\partial n}\right)^{2} \text { as } \varepsilon \rightarrow 0 .
$$

Moreover, it follows from $(3.1)^{\prime}$ that

$$
\int_{\partial B}\left(\frac{\partial G_{0}}{\partial n}\right)^{2} n=0
$$

To simplify the right-hand side of (3.13), we recall a result about the Green's function from [3, Theorem 4.3].

Lemma 3.6 Let $G(x, y)$ be the Green's function. Then for every $y \in B$,

$$
\begin{aligned}
\int_{\partial B}(x-y, n)\left(\frac{\partial G}{\partial n}(x, y)\right)^{2} d x & =-(N-2) g(y, y), \\
\int_{\partial B}\left(\frac{\partial G}{\partial n}(x, y)\right)^{2} n & =-\nabla g(y, y)
\end{aligned}
$$

where $n=n(x)$ denotes the outward normal to $\partial B$ at $x$ and $g$ is the regular part of the Green function.

Thus, setting $g_{0}=g(\cdot, 0)$, we can write $(3.13)$ as

$$
\varepsilon^{2(2-N)} \int_{\partial B}(x, n)\left(\frac{\partial \underline{u}_{\varepsilon}}{\partial n}\right)^{2} \rightarrow-J^{2}(N-2) g_{0}(0)=\frac{J^{2}}{\sigma_{N}},
$$

where we have used the explicit expression for $G_{0}$.

We now equate the estimates for respectively the left-hand and the right-hand side of the Pohozaev Identity (3.1), and we have that

$$
N \int_{\mathbf{R}^{N}} F(V(y)) d y=\frac{N-2}{2} \int_{\mathbf{R}^{N}} V(y) f(V(y)) d y .
$$

Moreover, it follows from (3.14), Lemma 3.6 that $\nabla g_{0}(0)=0$. This implies that 0 is a critical point of $g(x, x)$. This completes the proof of Theorem 3.1.

REMARKS 1. The conclusion of Theorem 3.1 is also true for the problem

$$
-\varepsilon^{2} \Delta u=u^{p}-C u^{q} \text { in } B, \quad u=0 \text { on } \partial B
$$

where $C>0, N(N-2)^{-1}<p<q<(N+2)(N-2)^{-1}$. In fact, it is known from [4] that there exists a small solution $\underline{u}_{\varepsilon}$ such that under the transformations: $y=\varepsilon^{-1} r$ 
and $v_{\varepsilon}(y)=\underline{u}_{\varepsilon}(r), v_{\varepsilon}(y) \rightarrow V$ in $C_{l o c}^{1}(0, \infty)$ as $\varepsilon \rightarrow 0$ (we can choose a subsequence if necessary) and $V$ is the unique positive (radial) solution of the problem

$$
-\Delta V=V^{p}-C V^{q} \text { in } \mathbf{R}^{N}, V(|x|) \rightarrow 0 \text { as }|x| \rightarrow \infty .
$$

The conclusion of Theorem 3.1 can be obtained by similar arguments to those in the proofs of Theorems 2.1 and 3.1.

2. We conjecture that the conclusion of Theorem 3.1 is still true for any $\rho>1$ in $\left(f_{2}\right)$. To prove this, we need to find a better estimate on $\underline{u}_{\varepsilon}$ as in Theorem 2.1. The behaviour of $f(s)$ near $s=0$ is useful for such an estimate.

3. If we consider (1.1) with $f$ satisfying $\left(f_{1}\right)-\left(f_{3}\right)$ and $\rho>1$ in $\left(f_{2}\right)$ on more general domains $\Omega$, we can find a small solution of (1.1) as a Mountain Pass Solution $\underline{u}_{\xi}$. We conjecture that when $\Omega$ is star-shaped, $\underline{u}_{\varepsilon}$ develops to a spike-layer solution at $x_{0} \in \Omega$ as $\varepsilon \rightarrow 0$, where $x_{0}$ is a critical point of the function $\phi(y)=g(y, y)$.

\section{REFERENCES}

[1] V. Benci and G. Cerami, 'Multiple positive solutions of some elliptic problems via the Morse theory and the domain topology', Calc. Var. Partial Differential Equations 2 (1994), 29-48.

[2] H. Berestycki and P.L. Lions, 'Nonlinear scalar field equations, I. Existence of a ground state', Arch. Rational Mech. Anal. 82 (1983), 313-345.

[3] H. Brezis and L.A. Peletier, 'Asymptotics for elliptic equations involving critical growth', in Partial Differential Equations and the Calcalus of Variations, (F. Colombini, A. Marino, L. Modica and S.Spagnolo, Editors) (Birkhäuser, Basel, 1989), pp. 149-192.

[4] E.N. Dancer, 'On positive solutions of some singularly perturbed problems where the nonlinearity changes sign', Topol. Methods Nonlinear Anal. 5 (1995), 141-175.

[5] B. Gidas, W.M. Ni and L. Nirenberg, 'Symmetry and related properties via the maximum principle', Comm. Math. Phys. 68 (1979), 209-243.

[6] M. Flucher and J. Wei, 'Asymptotic shape and location of small cores in elliptic free-boundary problems', Math. Z. 228 (1998), 683-703.

[7] J. Jang, 'On spike solutions of singularly perturbed semilinear Dirichlet problems', $J$. Diff. Equations 114 (1994), 370-395.

[8] F. Merle and L.A. Peletier, 'Asymptotic behaviour of positive solutions of elliptic equations with critical and supercritical growth I. The radial case', Arch. Rational Mech. Anal. 112 (1990), 1-19.

[9] W.M. Ni and J. Wei, 'On the location and profile of spike-layer solutions to singularly perturbed semilinear Dirichlet problems', Comm. Pure Appl. Math. 48 (1995), 731-768.

[10] W.M. Ni, I. Takagi and J. Wei, 'On the location and profile of spike-layer solutions to a singularly perturbed semilinear Dirichlet problems: Intermediate solutions', Duke Math. J. 94 (1998), 597-618.

Department of Mathematics

Henan Normal University

Xinxiang, 453002

Peoples Republic of China 